\title{
Digital Image Processing in CT Scan Facial Bone With Low mAs to Improve Image Quality and Radiation Dose Reduction
}

\author{
$* 1^{\text {st }}$ Widya Mufida \\ Faculty of Health Sciences \\ University of Aisyiyah Yogyakarta \\ Yogyakarta, Indonesia \\ widyamufida@unisayogya.ac.id
}

\author{
$2^{\text {nd }}$ Leny Latifah \\ Iodium Impaired Research and \\ Development Disorders (GAKI) \\ Magelang, Indonesia \\ lenylatifah@yahoo.com
}

\author{
$3^{\text {rd }}$ Jeffri Ardiyanto \\ Department of Radiodiagnostic and \\ Radioteraphy Technic \\ Semarang Health Polytechnic \\ Semarang, Indonesia \\ jeffri_ardiyanto@hotmail.com
}

\begin{abstract}
This study aims to get the lowest mAs on CT Scan Facial Bone so that the radiation dose can be reduced. The reduction of radiation dose can reduce image quality, so digital image processing applications are used which aim to improve image quality so as to produce image information that is still acceptable.
\end{abstract}

Keywords- mAs, slice thickness reconstruction, CT Scan Facial Bone

\section{INTRODUCTION}

In the CT scan there are several factors that affect the radiation dose including tube voltage $(\mathrm{kV})$, milliampersecond (mAs), sectional on thicness, pitch and distance of the tube to the CT scan isocenter [1,2] This factor is a complex combination that can affect the radiation dose, but the main factors in producing radiation are $\mathrm{kV}$ and $\mathrm{mAs} . \mathrm{kV}$ affects the amount of emission of $\mathrm{x}$-rays generated to penetrate an object, $\mathrm{kV}$ determine ma ksimum radiation bremstrahlung so the effect on the quality of x-ray radiation, while $\mathrm{mAs}$ affect the quantity of $\mathrm{x}$-ray radiation is generated, $\mathrm{mAs}$ proportional to the number of electrons that move from the cathode to the anode per unit time , mAs determine the number of photons or dose in a patient during scanning because the dose is directly proportional to mAs. In the image quality $\mathrm{kV}$ and $\mathrm{mAs}$ are the main factors that affect the contrast resolution and noise, while the spatial resolution is influenced by geometric factors [3].

Various attempts have been made by manufacturers to reduce the dose of CT Scan radiation such as tube current modulation and the use of low mAs. The mAs setting is the most common approach, when mAs are reduced by half the reduced radiation dose is around 50\% [4] but when mAs is reduced there is an increase in noise in the image. Filter back projection (FBP) is one filter that is often used to improve image quality by reducing noise, but FBP cannot produce consistent diagnostic image quality.

Another method used to reduce radiation doses is Iterative Reconstruction (IR). IR was first introduced in 2008, several clinical studies of this method can reduce noise and radiation doses by up to $50 \%$. Reconstruction methods on IR focus more on reducing the patient's dose, producing clearer images without reducing image [5]. IR capability is considered able to improve image quality and reduce radiation dose by maintaining diagnostic acceptability where non-ideal propagation data is reconstructed to be more significant than ordinary CT scans [6][7].

One of the checks that is often done is the CT Scan of Facial Bone or CT Scan of facial bones. In its application, some hospitals use the CT Scan head protocol and then do the reconstruction to get facial bone images during post scanning. In routine facial bone scan $\mathrm{CT}$ protocol alone the radiation dose produced $37 \mathrm{mGy}$ while the CT Scan head protocol resulting dose $60 \mathrm{mGy}$ [8] so that the radiation dose received by the patient is greater than it should be, concerns that occur due to the use of low doses will have an impact on image quality [9].

Efforts are made to reduce the radiation dose received by patients is to reduce $\mathrm{mAs}$, but by reducing $\mathrm{mAs}$ has an impact on decreasing contrast resolution, the smallest part of facial bone such as fine bone structure, joint gaps and paranasal sinuses that require thick, very thin slices do not seem so obvious due to increased noise [10], the combination of IR techniques with the CT Scan Facial Bone protocol is performed to reduce noise so as to display the smallest anatomical structure of bone. Some of the latest products from IR have special algorithms to reduce metal artifacts that can substantially reduce streak artifacts on teeth that use metal [11]. Reduction of radiation noise by using IR techniques with mAs that are too low cannot improve spatial resolution well.

Spasial esolution or detail can cause the boundaries between organ does not look stern or blur [12] then used filters unsharp mask to improve the spatial resolution of the image. Unsharp mask filter can improve the sharpness of borders organ by filtering the low frequency component and just passed high frequency components (high pass filter) so that the object is sharper than previously [3][13]

\section{METHOD}

This type of research is quantitative with a quasiexperimental design of pretest posttest control test to obtain anatomical image information with the lowest mAs or 
dose value. The research was carried out sequentially with variations in the value of mAs $(87.5 \mathrm{mAs}, 75 \mathrm{mAs}$, $62.5 \mathrm{mAs}, 50 \mathrm{mAs}$ and $37.5 \mathrm{mAs}$ ) and the application of an unsharp mask filter. The

study begins by scanning the facial bone object then using IR, after completion the image is reprocessed with a digital filter (unsharp mask). The object used is the anthhopomorphic phantom head.

Use of phant on antrhopomorphic head intended as a substitute for the patient's head props to avoid the risks do sis radiation $\mathrm{CT}$ scans given during the study.

Assessment of CT Scan Facial Bone anatomical information to get the lowest dose with optimal anatomical image information. The assessment was carried out by competent radiology specialists who have more than 5year experience in the interpretation of the CT Scan Facial Bone. The assessment was carried out by giving questionnaire sheets with the criteria assessed namely on coronal osteomeatal complex pieces on lamina cribrosa, ethmoid sinus, maxillary sinus, inferior rice concha, concha rice media, nasale septum, supraorbital incisors, lateral orbit and zygomaticum.

\section{RESULT}

In this study, the sample used was 5 variations of mAs as a treatment group, while the control group was $300 \mathrm{mAs}$. CTDIvol produced in the treatment group with $\mathrm{mAs} 87.5$ doses of radiation produced was $29.36 \mathrm{mGy}$, the dose with mAs 75 was $25.20 \mathrm{mGy}$, the dose with mAs 62.5 was 20.97 $\mathrm{mGy}$, the dose with mAs 50 was $16.78 \mathrm{mGy}$ and the dose with mAs 37.5 is $12.56 \mathrm{mGy}$, whereas in the control group $300 \mathrm{mAs}$ the resulting radiation dose is 53.66 .

TABLE I. SCAN PARAMETERS AND RADIATION DOSE WITH VARIATION IN MAS

\begin{tabular}{|c|c|c|c|}
\hline kV & mAs & Pitch & $\begin{array}{c}\text { CTDIvol } \\
(\mathbf{m G y})\end{array}$ \\
\hline \multicolumn{4}{|c|}{ Treatment group } \\
\hline 120 & 87.5 & 0.5 & 29.36 \\
120 & 75 & 0.5 & 25.20 \\
120 & 62.5 & 0.5 & 20.97 \\
120 & 50 & 0.5 & 16.78 \\
120 & 37.5 & 0.5 & 12.56 \\
\hline \multicolumn{4}{|c|}{ Control group } \\
\hline 120 & 300 & 0.5 & 53.66 \\
\hline
\end{tabular}

After obtaining the value of $\mathrm{mAs}$ or the lowest radiation dose with the image can still provide anatomical image information, then the application of an unsharp mask filter to improve the quality of the image decreases due to low mAs .

To determine differences in anatomical information on the variation of the value of mAs 37,5 with before and after application of unsharp mask filter on a CT scan Facial Bone test Wilcoxon test are shown in the table:
TABLE II. TEST THE DIFFERENCES IN ANATOMICAL IMAGE OF CT SCAN FACIAL BONE BEFORE AND AFTER THE FILTER OF THE UNSHARP MASK FILTER

\begin{tabular}{|l|c|}
\hline \multicolumn{1}{|c|}{ Variation } & p value \\
\hline $\begin{array}{l}\text { mAs 37.5 thick 1.5 } \\
\text { mm reconstruction slices before and after } \\
\text { applying the unsharp mask filter }\end{array}$ & 0.096 \\
\hline
\end{tabular}

The differences in anatomical image information before and after the application of filters unsharp mask. From the test results obtained variation of $\mathrm{mAs} 37,5$ with reconstruction slice thickness of $1.5 p$ value 0.096 ( $p>0.05)$, which means no image anatomical information difference before and after the application of filters unsharp mask. In addition to statistical calculations to see an increase in anatomical image information seen from the number of scoring results of respondents' assessments, in mAs 37.5 reconstructions of sliced thickness 1.5 before applying 62 and after applying to 69 .
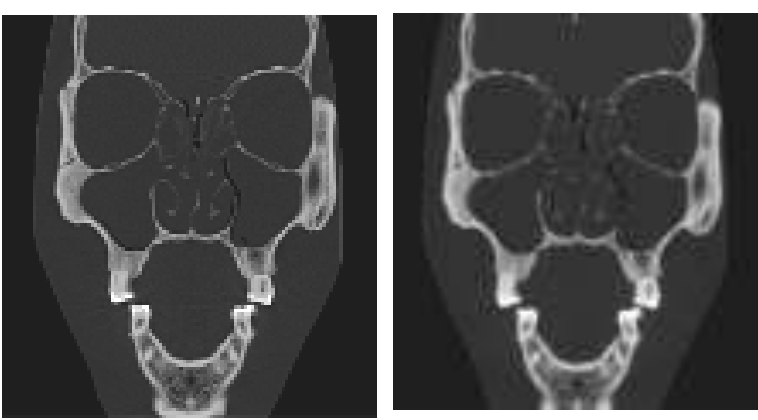

Fig. 1. Result of CT Scan Facial Bone radiograph with variation of $m A s$ 37,5 with reconstruction of $1,5 \mathrm{~mm}$ sliced thickness (a) without unsharp mask filter (b) with unsharpmask filter

\section{DISCUSSION}

The dose decreased with the decrease in given mAs value, obtained in the mAs control group used $150 \mathrm{mAs}$ with a radiation dose of $53.66 \mathrm{mGy}$, whereas in the highest $\mathrm{mAs}$ treatment group used was $87.5 \mathrm{mAs}$ with a dose of 29.36 mGy then the most mAs low at $37.5 \mathrm{mAs}$ at a dose of 12.56 mGy.

In this study there was a reduction in the dose in the treatment group with the lowest mAs compared to the control group which is a routine inspection protocol. mAs affect the quantity of x-rays produced in proportion to the number of electrons moving from the cathode to the anode per unit of time, mAs determine the number of photons (doses) in a patient during scanning because the dose is directly proportional to $\mathrm{mAs}$ [3], mAs are multiplications of tube currents $(\mathrm{mA})$ and time $(\mathrm{s})$ both influence noise and radiation dose then $\mathrm{mA}$ or $\mathrm{mAs}$ are considered to be the same [14].

In previous studies on CT Colonography examination using a routine protocol $50 \mathrm{mAs}$, using ASIR was able to reduce $50 \%$ of the routine dose to $25 \mathrm{mAs}$ without reducing image quality [15]. Other studies [6] reduced the radiation dose by reducing $\mathrm{mAs}$ by $35-60 \%$ from the initial protocol on the CT scan of the abdomen using $40 \%$ ASIR obtained the resulting dose based on CTDIvol is $12.5 \mathrm{mGy}$. 
The lowest radiation dose always has an impact on changes in the body's biological system, both at the molecular and cell level. When viewed from the radiation dose the effects of radiation are divided into stochastic and deterministic effects. The stochastic effect is an effect that occurs due to radiation exposure with a dose that causes cell changes, while the deterministic effect does not have a threshold dose that causes cell death due to radiation exposure to the body (ICRP). When radiation interacts with the body, the biological effects produced depend on the amount of radiation energy absorbed and the type of radiation. Children with cumulative doses of about $50 \mathrm{mGy}$ are three times the risk of leukemia, while radiation doses around $60 \mathrm{mGy}$ have a risk of brain cancer [16]. High risk factors of cumulative doses on CT scan are children under the age of 17 years [17]

When viewed from the concept of ALARA (As Low As Reasonably Achievable) by utilizing the lowest possible radiation by getting optimal results, one of them is the optimization of factors that can affect the radiation dose. Optimization refers to the lowest possible dose reduction by maintaining the image quality needed to make a diagnosis [3]. In this study, researchers optimized the value of mAs to obtain acceptable image results. The radiation dose produced based on routine protocol which is the factory setting is $53.66 \mathrm{mGy}$, while the results of measurements and calculations carried out radiation doses can be reduced to 12 , $56 \mathrm{mGy}$.

Some of the main factors that influence the visibility of images in displaying anatomical, tissue and pathological structures, namely the quality of the image that affects the anatomical image information. Image quality consists of contrast resolution, detail or spatial resolution, noise and artifacts that affect each other. One of the parameters that affect contrast resolution is $\mathrm{mAs}, \mathrm{mAs}$ is the multiplication of $\mathrm{mA}$ and $\mathrm{s}$ both affect the dose and contrast resolution [13]. In CT Resolution contrast scan is a characteristic of the imaging process that displays the differences between soft tissue and bone. Contrast resolution is directly proportional to $\mathrm{mAs}$ and inversely proportional to noise, when $\mathrm{mAs}$ is lowered then the resolution will decrease so that soft tissue cannot be clearly visualized because it is filled with noise, but objects with high density such as bones can still be visualized [18] even though spatial resolution or detail also decreases, the decrease in spatial resolution is not only caused by physical parameters such as the size of the focal spot and the dimensions of the detector element but during image reconstruction also affects the image results [19].

Iterative Reconstruction (IR) is one of the algorithms used to process images after data acquisition, IR performs a mathematical hybrid iteration process and statistical modeling to identify selectively reducing noise in an image [20]. IR repeatedly changes the Hounsfield pixel value of the image until the final value is found by using matrix algebra to change the value of each pixel (y) to the estimated new pixel value.

In this study IR was used to reduce doses and improve image quality because the given $\mathrm{mAs}$ were lower than routine protocols on the CT Scan Facial Bone examination. The type of IR used is Adaptive Statistical Iterative Reconstruction (ASIR) $60 \%$ is considered to be able to reduce noise and improve image quality, according to previous studies the use of ASIR below $50 \%$ does not significantly improve image quality with very low doses [21], whereas in other studies found ASIR levels at 20-40\% failed to show a significant difference in increasing signalto-ratio (SNR) compared to FBP, using ASIR 60\% and higher significantly could improve image quality [22] . According to the authors ASIR is often regarded as an effort to reduce radiation doses to patients indirectly, because ASIR is used to reconstruct a data created with a low exposure factor, so that a lot of noise that arises so it is done with repeated reconstruction.

One of the purposes of using IR in the CT Scan Facial Bone is to reduce noise so as to obtain maximum anatomic information. From the results of the anatomical image assessment on mAs 87.5 and mAs 75 with $1.5 \mathrm{~mm}$ slice thick reconstruction have the best image information because it can display the anatomical structure clearly and have sharp contours this is evidenced by a total score of 62 .

Matlab image processing with unsharp mask filter is a common method used to improve image results, especially to improve image quality due to a decrease in spatial resolution (CT), unsharp mask pis pens can be used easily in filtering in real time [13]. Determination of the image to be processed by unsharp mask filter by considering the variable dose of radiation produced and image information can still be accepted. At mAs 37.5, the 5 radiation doses produced were 12.56 with $1.5 \mathrm{~mm}$ slice thickness scoring the obtained image information 62. In addition, the anatomical information variable consideration was due to the aforementioned radiographs that had the authority to interpret the radiology specialist.

The results of research on increasing anatomic information after applying the unsharp mask filter can be concluded that there was an increase in anatomic information in $\mathrm{mA}$ s 37.5 with reconstruction of 1.5 sliced thickness although statistically $37.5 \mathrm{mAs}$ with reconstruction of 1.5 sliced thickness did not have a significant difference. between before and after applying the unsharp mask, but in a qualitative manner there was an increase in the score from 62 to 69. In addition, an increase in image information can be seen in the mean rank with mAs 37,5 reconstructions of 1.5 slice thickness from 2.39 to 2.86 .

Spatial resolution or detail in an image affects the visibility of the smallest object and the edge of the organ because it gives a blur effect on the image. Spatial resolution is not only influenced by physical parameters such as the size of the focal spot and the dimensions of the detector element but is influenced by other factors during reconstruction such as the use of IR or filtered back projection ( FBP) (Spatial Resolution in CT) besides the sliced thickness reconstruction which is one of factors that affect detail.

CT facial facial bone images consisting of bone and soft tissues in the paranasal sinuses certainly require high spatial resolution to display the smallest parts so as to distinguish between anatomical and pathological structures in the bone. With the application of unsharp maskk detail on the bone increases, the brick edge of the organ becomes sharper, the sutures formed because the meeting between bones can be seen clearly so as to distinguish between the fissure which is pathology and fissure which is the suture. In the $1.5 \mathrm{~mm}$ slice 
thickness reconstruction the results of the assessment using a statistical test do not have a significant difference, this is because the thin slice thickness reconstruction has good spatial detail or resolution [12], but from the total score obtained before and after setting The application of the Unsharp Mask filter has increased by 7 points.

Several previous studies used unsharp mask filters on incephalometric radiographic images to show the smallest visibilities structure characteristic of bone that is bright [23]. The use of unsharp mask filters on medical images can sharpen the edges of the anatomy of organs, increasing the edge contrast in more detail so that the trabecular pattern can be seen clearly [24].

Based on the results of the above study, it can be concluded that the optimal mAs value after application in the examination of the optimal Facial Bone CT Scan is mAs 37.5.

\section{CONCLUSION}

The radiation dose decreases with decreasing mAs given. The lowest mAs value of $37.5 \mathrm{mAs}$ with slices of 2.5 and 1.5 $\mathrm{mm}$ have image information that can still be visualized but the contour border is less sharp. There is an increase in anatomic information before and after the application of the unsharp mask filter on the CT Scan Facial Bone.

\section{REFERENCES}

[1] Zacharias, C., Alessio, A. M., Otto, R. K., Iyer, R. S., Philips, G. S., Swanson, J. O., \& Thapa, M. M.. Pediatric CT: Strategies to lower radiation dose. American Journal of Roentgenology. http://doi.org/10.2214/AJR.12.9026, 2013.

[2] Andriani, i., Anam, c., \& Setiawati, e. Penentuan Ct Dose Index (CTDI) Untuk Variasi Slice Thickness Dengan Program Dosxyznrc. Berkala Fisika, 15(3), 69-76, 2012.

[3] Seeram E. Computed Tomography Physical Principles, Clinical Applications and Quality Control. Vol Fourth Ed. St Louis Missouri;2016

[4] Kalra, M. K., Woisetschläger, M., Dahlström, N., Singh, S. Lindblom, M., Choy, G., Persson, A. Radiation dose reduction with sinogram affirmed iterative reconstruction technique for abdominal computed tomography. Journal of Computer Assisted Tomography, 36(3), 339-346. http://doi.org/10.1097/RCT.0b013e31825586c0, 2012.

[5] Brady, S. L., Yee, B. S., \& Kaufman, R. A. Characterization of adaptive statistical iterative reconstruction algorithm for dose reduction in CT: A pediatric oncology perspective. Medical Physics, 39(9), 5520-5531. http://doi.org/10.1118/1.4745563, 2012.

[6] Hara, A. K., Paden, R. G., Silva, A. C., Kujak, J. L., Lawder, H. J., \& Pavlicek, W. Iterative reconstruction technique for reducing body radiation dose at CT: Feasibility study. American Journal of Roentgenology, 193(3), http://doi.org/10.2214/AJR.09.2397, 2009

[7] Löve, A., Olsson, M. L., Siemund, R., Stålhammar, F., BjörkmanBurtscher, I. M., \& Söderberg, M. Six iterative reconstruction algorithms in brain CT: a phantom study on image quality at different radiation dose levels. The British journal of radiology, 86(1031), 20130388, 2013.

[8] Smith-Bindman, R., Moghadassi, M., Wilson, N., Nelson, T.R., Boone, J.M., Cagnon, C.H., Gould, R., Hall, D.J., Krishnam, M., Lamba, R. and McNitt-Gray, M., Radiation doses in consecutive CT examinations from five University of California Medical Centers. Radiology, 277(1), pp.134-141, 2015.
[9] Smith-Bindman, R., Lipson, J., Marcus, R., Kim, K.P., Mahesh, M., Gould, R., De González, A.B. and Miglioretti, D.L.,. Radiation dose associated with common computed tomography examinations and the associated lifetime attributable risk of cancer. Archives of internal medicine, 169(22), pp.2078-2086, 2009

[10] Singh, S., Kalra, M. K., Hsieh, J., Licato, P. E., Do, S., Pien, H. H., \& Blake, M. A. Abdominal CT: comparison of adaptive statistical iterative and filtered back projection reconstruction techniques. Radiology, 257(2), 373-383, 2010.

[11] Singh, S., Kalra, M.K., Gilman, M.D., Hsieh, J., Pien, H.H., Digumarthy, S.R. and Shepard, J.A.O. Adaptive statistical iterative reconstruction technique for radiation dose reduction in chest CT: a pilot study. Radiology, 259(2), pp.565-573, 2011.

[12] Kalender, W.A. X-ray computed tomography. Physics in Medicine \& Biology, 51(13), p.R29, 2006

[13] Ahmed HS, Nordin MJ. Improving diagnostic viewing of medical images using enhancement algorithms. Journal of Computer Science. 2011;7(12):1831.

[14] Goldman, L. W. Principles of CT: radiation dose and image quality. Journal of Nuclear Medicine Technology, 35(4), 213-225-228. https://doi.org/10.2967/jnmt.106.037846, 2007

[15] Flicek, K.T., Hara, A.K., Silva, A.C., Wu, Q., Peter, M.B. and Johnson, C.D. Reducing the radiation dose for CT colonography using adaptive statistical iterative reconstruction: a pilot study. American Journal of Roentgenology, 195(1), pp.126-131, 2010.

[16] Pearce, M. S., Salotti, J. A., Little, M. P., McHugh, K., Lee, C., Kim, K. P., ... De González, A. B. Radiation exposure from CT scans in childhood and subsequent risk of leukaemia and brain tumours: A retrospective cohort study. The Lancet, 380(9840), 499- 505. http://doi.org/10.1016/S0140-6736(12)60815-0, 2012.

[17] Allen, B.C., Baker, M.E., Einstein, D.M., Remer, E.M., Herts, B.R., Achkar, J.P., Davros, W.J., Novak, E. and Obuchowski, N.A., Effect of altering automatic exposure control settings and quality reference $\mathrm{mAs}$ on radiation dose, image quality, and diagnostic efficacy in MDCT enterography of active inflammatory Crohn's disease. American Journal of Roentgenology, 195(1), pp.89-100, 2010.

[18] Sprawls Education Fondation. Physical Prinsiples of Medical Imaging (http://www.sprawls.org/resources, diakses tanggal 14 Agustus 2018)

[19] Spatial Resolution in CT, Journal of the International Commission on Radiation Units and Measurements, Volume 12, Issue 1, 1 April 2012,

[20] Pages 107-120, https://doi.org/10.1093/jicru/ndt001

[21] Geyer, L. L., Schoepf, U. J., Meinel, F. G., Nance, J. W., Bastarrika, G., Leipsic, J. A., De Cecco, C. N. State of the Art: Iterative CT Reconstruction Techniques. Radiology, 276(2), 339-357. https://doi.org/10.1148/radiol.2015132766, 2015.

[22] Widmann, G., Schullian, P., Gassner, E. M., Hoermann, R., Bale, R., \& Puelacher, W. Ultralow-dose CT of the craniofacial bone for navigated surgery using adaptive statistical iterative reconstruction and model-based iterative reconstruction: 2D and 3D image quality. American Journal of Roentgenology, 204(3), 563-569. http://doi.org/10.2214/AJR.14.12766, 2015.

[23] Rapalino, O., Kamalian, S., Kamalian, S., Payabvash, S., Souza, L. C. S., Zhang, D., ... Pomerantz, S. R. Cranial CT with Adaptive Statistical Iterative Reconstruction: Improved Image Quality with Concomitant Radiation Dose Reduction. American Journal of Neuroradiology, 33(4), 609-615. http://doi.org/10.3174/ajnr.A2826, 2012.

[24] Hoeffner EG, Quint DJ, Peterson B et al: Development of a protocol for coronal reconstruction of the maxillofacial region from axial helical CT data. BJR, 2001; 74: 323-27

[25] Sen, A., L. Lan and K.R. Hoffmann. Improved coronary vessel tracking using non-linear filter enhanced X-ray angiograms. Proceedings of the 19th Annual International Conference on Engineering Medicine Biology Society, Oct. 30- Nov. 2, IEEE XPlore Press, Chicago, II, USA. pp: 573-576. DOI: 10.1109/IEMBS.1997.757674, 2002. 